\title{
RITZ VALUE LOCALIZATION FOR NON-HERMITIAN MATRICES*
}

\author{
RUSSELL L. CARDEN ${ }^{\dagger}$ AND MARK EMBREE ${ }^{\dagger}$
}

\begin{abstract}
Rayleigh-Ritz eigenvalue estimates for Hermitian matrices obey Cauchy interlacing, which has helpful implications for theory, applications, and algorithms. In contrast, few results about the Ritz values of non-Hermitian matrices are known, beyond their containment within the numerical range. To show that such Ritz values enjoy considerable structure, we establish regions within the numerical range in which certain Ritz values of general matrices must be contained. To demonstrate that localization occurs even for extreme examples, we carefully analyze possible Ritz value combinations for a three-dimensional Jordan block.
\end{abstract}

Key words. Ritz values, numerical range, inverse field of values problem

AMS subject classifications. 15A18, 15A42, 47A10, 65F 15

DOI. $10.1137 / 120872693$

1. Introduction. Rayleigh-Ritz eigenvalue estimates arise throughout applied mathematics, facilitating the analysis of physical systems and enabling a variety of computational algorithms. For example, iterative methods for solving large linear systems and eigenvalue problems rely fundamentally on Ritz values and their harmonic variants. One cannot fully comprehend the behavior of these algorithms, nor see how best to accelerate their convergence, without deeply understanding Ritz values.

Consider a matrix $\mathbf{A} \in \mathbb{C}^{n \times n}$ and a $p$-dimensional subspace $\mathcal{V} \subset \mathbb{C}^{n}$, and let the columns of $\mathbf{V} \in \mathbb{C}^{n \times p}$ form an orthonormal basis for $\mathcal{V}$. Hence $\operatorname{Ran}(\mathbf{V})=\mathcal{V}$, where $\operatorname{Ran}(\cdot)$ denotes the range (column space) of a matrix. The eigenvalues of $\mathbf{V}^{*} \mathbf{A V}$ are called the Ritz values of $\mathbf{A}$ with respect to $\mathcal{V}$. These values are independent of the orthonormal basis $\mathbf{V}$ for $\mathcal{V}$.

After more than a century of study much is known about the Ritz values of Hermitian matrices (and self-adjoint operators in Hilbert space). Among the earliest and most descriptive results for matrices is the Cauchy interlacing theorem; see, e.g., [15]. Suppose $\mathbf{A}$ is Hermitian with eigenvalues $\lambda_{1} \leq \lambda_{2} \leq \cdots \leq \lambda_{n}$. Then $\mathbf{V}^{*} \mathbf{A V}$ is Hermitian, so its eigenvalues - the Ritz values of $\mathbf{A}$ with respect to $\mathcal{V}$-are also real: $\theta_{1} \leq \theta_{2} \leq \cdots \leq \theta_{p}$. The Cauchy interlacing theorem gives

$$
\theta_{k} \in\left[\lambda_{k}, \lambda_{n+k-p}\right], \quad k=1, \ldots, p .
$$

Beyond the Hermitian case, our understanding remains surprisingly primitive. Recent work provides insight for normal matrices, including a geometric description of the Ritz values for $p=n-1[5,13]$, and a characterization of Ritz values from Krylov subspaces [2]. For general matrices, little has been said beyond the tautology that all Ritz values must fall within the numerical range (field of values)

$$
W(\mathbf{A}):=\left\{\mathbf{v}^{*} \mathbf{A} \mathbf{v}: \mathbf{v} \in \mathbb{C}^{n},\|\mathbf{v}\|=1\right\},
$$

which is simply the set of all Ritz values. For $p=1$, several recently proposed algorithms identify subspaces that generate any given $\theta_{1} \in W(\mathbf{A})$, the so-called inverse

\footnotetext{
*Received by the editors April 9, 2012; accepted for publication (in revised form) by J. Barlow September 26, 2012; published electronically December 12, 2012. Supported by National Science Foundation grant DMS-CAREER-0449973.

http://www.siam.org/journals/simax/33-4/87269.html

${ }^{\dagger}$ Department of Computational and Applied Mathematics, Rice University, Houston, TX 770051892 (Russell.L.Carden@rice.edu, embree@rice.edu).
} 
field of values problem $[3,6,20]$. For subspaces of dimension $p>1$ this problem is much more difficult; indeed, given two points $\theta_{1}, \theta_{2} \in W(\mathbf{A})$, no satisfactory method is known to verify whether there exists any two-dimensional subspace $\mathcal{V} \subset \mathbb{C}^{n}$ that gives both $\theta_{1}$ and $\theta_{2}$ as Ritz values. In general, the problem of identifying those sets $\left\{\theta_{1}, \ldots, \theta_{p}\right\} \subset W(\mathbf{A})$ that can be realized as Ritz values from a $p$-dimensional subspace, along with that generating subspace, is known as the $i F O V(p)$ problem [3]. We seek to understand this problem for $2 \leq p \leq n-1$. Absent such insight, we can summarize the state of the art as follows: little, if anything, is known about the "inner geometry" [20] of the numerical range for nonnormal A.

This situation has unfortunate consequences, complicating eigenvalue estimation for non-self-adjoint operators (as motivated by problems in physics and engineering) and preventing a deep understanding of iterative methods for large-scale linear systems and eigenvalue problems. Indeed the latter motivated our present study. We wish to analyze the convergence of Sorensen's implicitly restarted Arnoldi algorithm [19], a leading method for computing eigenvalues of large, sparse matrices that is implemented in the ARPACK software package [12] and MATLAB's eigs command. This algorithm develops approximations to invariant subspaces of $\mathbf{A}$ from Krylov subspaces whose starting vectors are repeatedly refined through application of a filter polynomial. The standard "exact shift" procedure identifies the Ritz values that most closely resemble the desired eigenvalues (e.g., the rightmost eigenvalues), then uses the remaining Ritz values as roots of the filter polynomial. This process will fail when one of these roots coincides with a desired eigenvalue, effectively deflating that eigenvalue from the approximating subspace [7]. A satisfactory convergence theory that accounts for such cases must rely on fine properties of the Ritz values.

This work began with an experiment that precisely illustrates how some generalization of "interlacing" - that is, a geometric restriction on the location of certain Ritz values - can hold even for the antithesis of the well-understood Hermitian case. Take $\mathbf{A}$ to be the $3 \times 3$ Jordan block

$$
\mathbf{A}=\left[\begin{array}{lll}
0 & 1 & 0 \\
0 & 0 & 1 \\
0 & 0 & 0
\end{array}\right]
$$

It is well known that $W(\mathbf{A})=\{z \in \mathbb{C}:|z| \leq \sqrt{2} / 2\}$, the closed disk of radius $\sqrt{2} / 2$ in the complex plane, centered at the origin $[8$, p. 9$]$. Now generate random twodimensional (complex) subspaces, compute the Ritz values, and sort them by their real parts. Figure 1.1 illustrates the results: the leftmost Ritz values appear to cover only a portion of the numerical range. In none of these 10,000 experiments does the leftmost Ritz value fall near the rightmost extent of $W(\mathbf{A})$; for example, it appears to be impossible for both Ritz values to fall near the point $z=1 / 2$.

This observation is easy to confirm analytically, at least in a coarse manner. Let $\mathcal{V}$ be a two-dimensional subspace of $\mathbb{C}^{3}$ that is spanned by the orthonormal basis $\left\{\mathbf{v}_{1}, \mathbf{v}_{2}\right\}$. Construct the matrix $\mathbf{V}=\left[\mathbf{v}_{1}, \mathbf{v}_{2}\right]$, and let $\mathbf{v}_{3}$ be a unit vector orthogonal to $\mathcal{V}$, so that $\mathbf{U}=\left[\mathbf{v}_{1}, \mathbf{v}_{2}, \mathbf{v}_{3}\right] \in \mathbb{C}^{3 \times 3}$ is unitary and the eigenvalues of $\mathbf{V}^{*} \mathbf{A V}$ are the Ritz values $\theta_{1}$ and $\theta_{2}$ of $\mathbf{A}$ from $\mathcal{V}$. Letting $\operatorname{tr}(\cdot)$ denote the trace, notice that

$$
0=\operatorname{tr}(\mathbf{A})=\operatorname{tr}\left(\mathbf{U}^{*} \mathbf{A} \mathbf{U}\right)=\operatorname{tr}\left(\mathbf{V}^{*} \mathbf{A V}\right)+\mathbf{v}_{3}^{*} \mathbf{A} \mathbf{v}_{3}=\theta_{1}+\theta_{2}+\mathbf{v}_{3}^{*} \mathbf{A} \mathbf{v}_{3} .
$$

Label the leftmost Ritz value as $\theta_{1}$, so $\operatorname{Re}\left(\theta_{1}\right) \leq \operatorname{Re}\left(\theta_{2}\right)$. Since $\mathbf{v}_{3}^{*} \mathbf{A} \mathbf{v}_{3} \in W(\mathbf{A})$,

$$
\operatorname{Re}\left(\theta_{1}\right)=-\operatorname{Re}\left(\mathbf{v}_{3}^{*} \mathbf{A} \mathbf{v}_{3}\right)-\operatorname{Re}\left(\theta_{2}\right) \leq \frac{\sqrt{2}}{2}-\operatorname{Re}\left(\theta_{1}\right),
$$



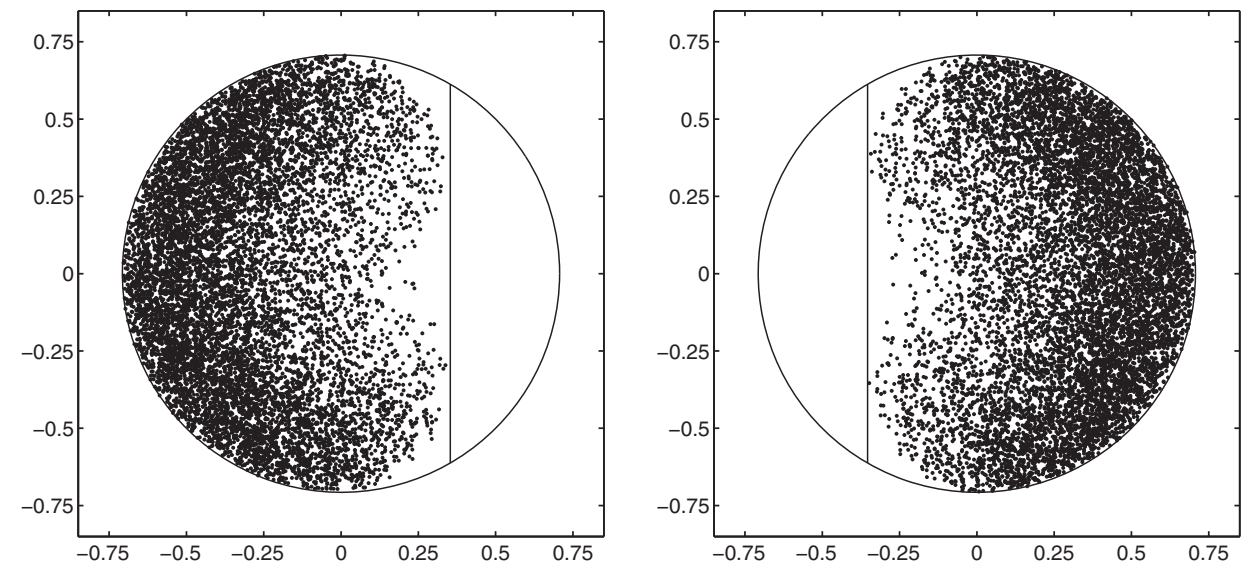

FIG. 1.1. Ritz values drawn from 10,000 random two-dimensional subspaces of $\mathbb{C}^{3}$. Each pair of Ritz values is sorted by real part: the leftmost Ritz value from each experiment is shown on the left, while the rightmost Ritz value is plotted on the right. In both cases the solid circle denotes the boundary of $W(\mathbf{A})$, while the vertical lines indicate the upper bound on the leftmost Ritz value (1.2) and the analogous lower bound on the rightmost Ritz value.

from which we conclude that

$$
\operatorname{Re}\left(\theta_{1}\right) \leq \frac{\sqrt{2}}{4}
$$

This bound and the analogous lower bound $-\sqrt{2} / 4 \leq \operatorname{Re}\left(\theta_{2}\right)$ are shown as vertical lines in Figure 1.1.

In the spirit of these simple bounds, we establish in the next section containment regions that "localize" the Ritz values of general matrices. While not as sharp as Cauchy interlacing for Hermitian matrices, these bounds do reveal considerable "inner geometry" within the numerical range. We later give more detailed analysis for $p=2$ Ritz values of a $3 \times 3$ Jordan block, which reveals the additional structure hinted at in Figure 1.1 and indicates the challenge of completely understanding Ritz values for general matrices. To the best of our knowledge, this is the first work to precisely analyze the Ritz values of any nonnormal matrix.

2. Ritz values of general matrices. The simple bound (1.2) on the rightmost extent of the leftmost Ritz value for a three-dimensional Jordan block, derived using a trace argument, is a special case of more general analysis based on eigenvalue majorization. In this section, we develop bounds on the Ritz values, sorted by real part and magnitude. Such bounds are useful for stability analysis of dynamical systems, where one seeks rightmost eigenvalues for continuous time systems and largest magnitude eigenvalues for discrete time systems. For similar bounds on the phases of Ritz values, see [4, section 3.2.2].

2.1. Bounds on the real part of Ritz values. Any matrix $\mathbf{A} \in \mathbb{C}^{n \times n}$ can be decomposed into the sum of its Hermitian and skew-Hermitian parts, $\mathbf{H}:=\left(\mathbf{A}+\mathbf{A}^{*}\right) / 2$ and $\mathbf{S}:=\left(\mathbf{A}-\mathbf{A}^{*}\right) / 2 i$; some call $\mathbf{A}=\mathbf{H}+i \mathbf{S}$ the Cartesian decomposition [17]. We wish to study the Ritz values of $\mathbf{A}$ drawn from the $p$-dimensional subspace $\operatorname{Ran}(\mathbf{V})$, where $\mathbf{V} \in \mathbb{C}^{n \times p}$ has orthonormal columns. Without loss of generality, assume this basis is chosen in such a way that $\mathbf{V}^{*} \mathbf{A V}$ is upper triangular (via the Schur decomposition), and hence the Ritz values are on its main diagonal. Label them by increasing 
real part: $\operatorname{Re} \theta_{1} \leq \operatorname{Re} \theta_{2} \leq \cdots \leq \operatorname{Re} \theta_{p}$. Let the columns of $\widehat{\mathbf{V}} \in \mathbb{C}^{n \times(n-p)}$ form an orthonormal basis for the orthogonal complement of $\operatorname{Ran}(\mathbf{V})$, which can always be done in a manner that makes $\widehat{\mathbf{V}}^{*} \mathbf{A} \widehat{\mathbf{V}} \in \mathbb{C}^{(n-p) \times(n-p)}$ upper triangular. Label the eigenvalues of $\widehat{\mathbf{V}}^{*} \mathbf{A} \widehat{\mathbf{V}}$ as $\theta_{p+1}, \ldots, \theta_{n}$. The set $\Theta:=\left\{\theta_{1}, \theta_{2}, \ldots, \theta_{n}\right\}$ comprises the diagonal entries of $[\mathbf{V} \widehat{\mathbf{V}}]^{*} \mathbf{A}[\mathbf{V} \widehat{\mathbf{V}}]$, while the real parts $\operatorname{Re} \theta_{1}, \operatorname{Re} \theta_{2}, \ldots, \operatorname{Re} \theta_{n}$ are the diagonal entries of $[\mathbf{V} \widehat{\mathbf{V}}]^{*} \mathbf{H}[\mathbf{V} \widehat{\mathbf{V}}]$.

Now let $\mu_{1} \leq \mu_{2} \leq \cdots \leq \mu_{n}$ denote the eigenvalues of $\mathbf{H}$, and relabel the members of $\Theta$ by increasing real part: $\operatorname{Re} \theta_{(1)} \leq \operatorname{Re} \theta_{(2)} \leq \cdots \leq \operatorname{Re} \theta_{(n)}$. By a classical result of Schur [1, p. 35], the vector $\left[\operatorname{Re} \theta_{(j)}\right]_{j=1}^{n}$ of (ordered) diagonal entries of $[\mathbf{V ~} \widehat{\mathbf{V}}]^{*} \mathbf{H}[\mathbf{V} \widehat{\mathbf{V}}]$ majorizes the vector $\left[\mu_{j}\right]_{j=1}^{n}$ of eigenvalues, i.e.,

$$
\sum_{j=1}^{k} \mu_{j} \leq \sum_{j=1}^{k} \operatorname{Re} \theta_{(j)}, \quad k=1, \ldots, n,
$$

with equality for $k=n$. Since $\operatorname{Re} \theta_{(j)} \leq \operatorname{Re} \theta_{j}$, we have

$$
\sum_{j=1}^{k} \mu_{j} \leq \sum_{j=1}^{k} \operatorname{Re} \theta_{j}, \quad k=1, \ldots, p,
$$

which means that the vector $\left[\operatorname{Re} \theta_{j}\right]_{j=1}^{p}$ weakly majorizes the vector $\left[\mu_{j}\right]_{j=1}^{p}$. From this majorization one can derive bounds that localize where the Ritz values $\theta_{j}$ of $\mathbf{A}$ must fall in the complex plane. For example, the weak majorization (2.1) with $k=2$ implies

$$
\mu_{1}+\mu_{2} \leq \operatorname{Re} \theta_{1}+\operatorname{Re} \theta_{2} \leq 2 \operatorname{Re} \theta_{2},
$$

and so

$$
\frac{\mu_{1}+\mu_{2}}{2} \leq \operatorname{Re} \theta_{2},
$$

restricting the leftmost extent of the second Ritz value of $\mathbf{A}$. For the $k$ th Ritz value,

$$
\mu_{1}+\cdots+\mu_{k} \leq \operatorname{Re} \theta_{1}+\cdots+\operatorname{Re} \theta_{k} \leq k \operatorname{Re} \theta_{k} .
$$

Applying the analysis to $-\mathbf{A}$ yields

$$
\mu_{n-p+k}+\cdots+\mu_{n} \geq \operatorname{Re} \theta_{k}+\cdots+\operatorname{Re} \theta_{p} \geq(p-k+1) \operatorname{Re} \theta_{k} .
$$

These bounds are summarized in the following theorem. The idea of majorizing the real part of the spectrum by the spectrum of $\mathbf{H}$ dates back to Ky Fan in the 1950s [1, Proposition III.5.3], [14, section 9.F], though we are unaware of previous use of this fact to bound Ritz values.

Theorem 2.1. Let $\theta_{1}, \ldots, \theta_{p}$ denote the Ritz values of $\mathbf{A} \in \mathbb{C}^{n \times n}$ drawn from a $p<n$ dimensional subspace, labeled by increasing real part: $\operatorname{Re} \theta_{1} \leq \cdots \leq \operatorname{Re} \theta_{p}$. Then for $k=1, \ldots, p$,

$$
\frac{\mu_{1}+\cdots+\mu_{k}}{k} \leq \operatorname{Re} \theta_{k} \leq \frac{\mu_{n-p+k}+\cdots+\mu_{n}}{p-k+1},
$$

where $\mu_{1} \leq \cdots \leq \mu_{n}$ are the eigenvalues of $\mathbf{H}=\frac{1}{2}\left(\mathbf{A}+\mathbf{A}^{*}\right)$. 
Corollary 2.2. At most $m<p$ Ritz values of $\mathbf{A} \in \mathbb{C}^{n \times n}$ from a $p$-dimensional subspace can be contained in each of the following subsets of the complex plane:

$$
\begin{aligned}
& \Omega_{\ell, m}:=\left\{z \in W(\mathbf{A}): \operatorname{Re} z \in\left[\ell_{m}, \ell_{m+1}\right)\right\}, \\
& \Omega_{r, m}:=\left\{z \in W(\mathbf{A}): \operatorname{Re} z \in\left(r_{m+1}, r_{m}\right]\right\},
\end{aligned}
$$

where, for $k=1, \ldots, p$,

$$
\ell_{k}:=\frac{\mu_{1}+\cdots+\mu_{k}}{k}, \quad r_{k}:=\frac{\mu_{n-k+1}+\cdots+\mu_{n}}{k} .
$$

For $k=1$ and $k=p,(2.3)$ yields the trivial statement

$$
\mu_{1} \leq \operatorname{Re} \theta_{1} \leq \operatorname{Re} \theta_{p} \leq \mu_{n},
$$

which more directly follows from the fact that Ritz values are in the numerical range, and $\operatorname{Re}(W(\mathbf{A}))=\left[\mu_{1}, \mu_{n}\right]$. For $k \in\{2, \ldots, p-1\}$, the theorem can provide considerable insight into the interior structure of the numerical range. We shall examine this in more detail for Jordan blocks at the end of this section.

Is Theorem 2.1 sharp? If $\mathbf{A}$ is Hermitian, then $\mathbf{H}=\mathbf{A}$, and $\mu_{1}, \ldots, \mu_{n}$ are the eigenvalues of $\mathbf{A}$. One can immediately compare Theorem 2.1 to the bounds from the Cauchy interlacing theorem:

$$
\mu_{k} \leq \theta_{k} \leq \mu_{n-p+k} .
$$

The Cauchy bounds, which can always be attained, will be considerably tighter than Theorem 2.1 when the eigenvalues of $\mathbf{A}=\mathbf{H}$ are well separated.

The slack in Theorem 2.1 can be attributed to the second inequality in (2.2), for the majorization in (2.1) becomes strong (i.e., with equality for $k=p$ ), when the subspace $\operatorname{Ran}(\mathbf{V})$ corresponds to the eigenspace for the $p$ smallest eigenvalues of $\mathbf{H}$. If the eigenvalues of the Hermitian part are distinct, the corresponding subspaces are unique. ${ }^{1}$ To obtain sharper bounds, one could draw in further information about the numerical range, e.g., based on the skew-Hermitian part of A. (Recently Psarrakos and Tsatsomeros have used the second largest eigenvalue of $\mathbf{H}$ to develop inclusion regions for the spectrum [16].)

2.2. Illustration: Jordan blocks. When $\mathbf{A}$ is an $n$-dimensional Jordan block (ones on the first superdiagonal, zeros elsewhere), we can compute the bounds in Theorem 2.1 explicitly. In this case

$$
\mathbf{H}=\frac{1}{2}\left[\begin{array}{cccc}
0 & 1 & & \\
1 & 0 & \ddots & \\
& \ddots & \ddots & 1 \\
& & 1 & 0
\end{array}\right]
$$

has well-known eigenvalues

$$
\mu_{j}=\cos \left(\frac{(n-j+1) \pi}{n+1}\right), \quad j=1, \ldots, n ;
$$

\footnotetext{
${ }^{1}$ For the $n=3$ Jordan block, this is precisely why the left plot in Figure 1.1 suggests that only two points (complex conjugates) might attain the bound $\operatorname{Re} \theta_{1} \leq \sqrt{2} / 4$. In this case, take $\operatorname{Ran}(\mathbf{V})$ to be the eigenspace of $\mathbf{H}$ corresponding to $\mu_{2}$ and $\mu_{3}$. Then $W\left(\mathbf{V}^{*} \mathbf{A V}\right)$ is an ellipse (since $\mathbf{V}^{*} \mathbf{A V} \in \mathbb{C}^{2 \times 2}$; see $[10$, section 1.3$\left.]\right)$ with minor axis $[0, \sqrt{2} / 2]=\left[\mu_{2}, \mu_{3}\right]$. The Ritz values are $(\sqrt{2} \pm \sqrt{2} i) / 4$, attaining the bound $\operatorname{Re} \theta_{1} \leq \sqrt{2} / 4$.
} 


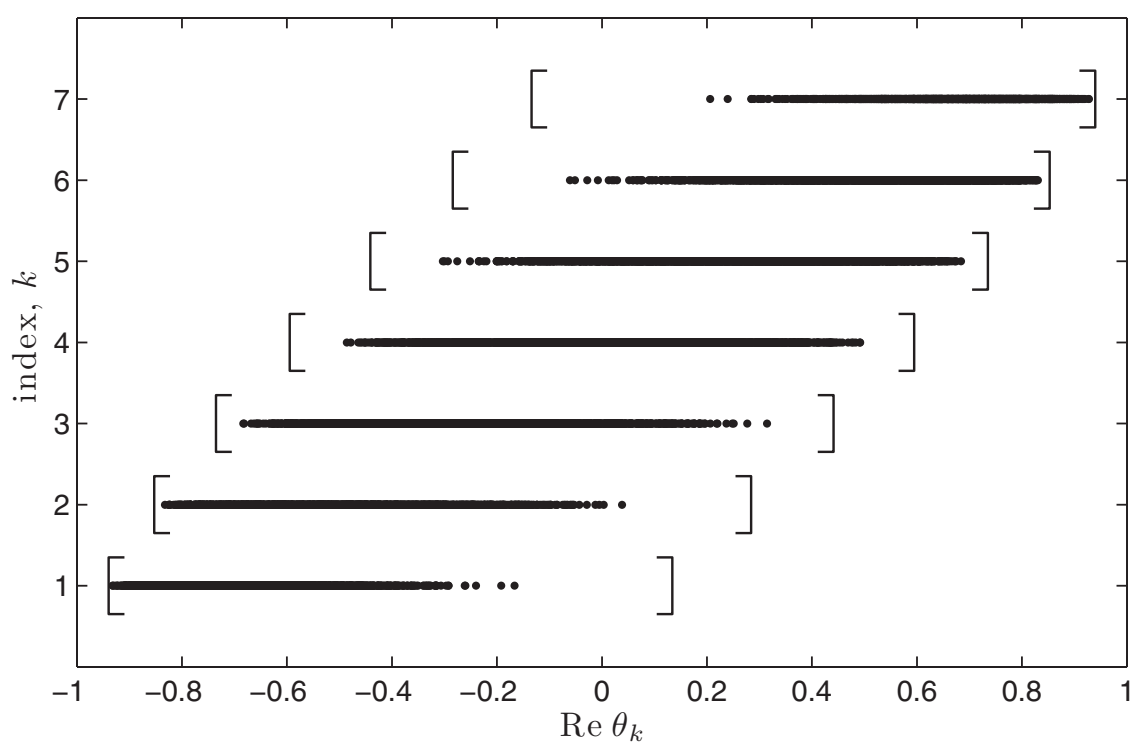

FIG. 2.1. Theorem 2.1 illustrated for a Jordan block of dimension $n=8$. For each of $k=$ $1, \ldots, n-1$, the bound from Theorem 2.1 is shown as a bracket containing the real parts of the Ritz values $\theta_{k}$ drawn from 2000 random real $p=n-1$ dimensional subspaces (solid black dots).

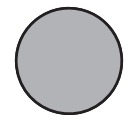

$n=2$

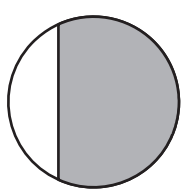

$n=4$

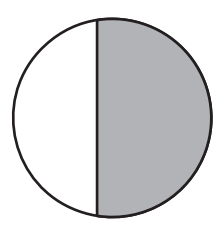

$n=8$

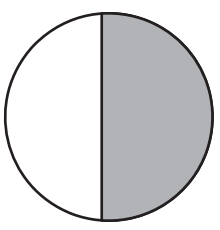

$n=16$

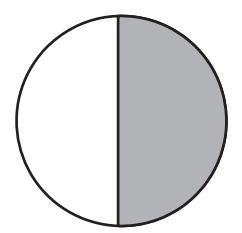

$n=32$

FIG. 2.2. Containment regions (gray) for the rightmost eigenvalue $\theta_{n-1}$ of an $n \times n$ Jordan block drawn from a $p=n-1$ dimensional subspace, based on Theorem 2.1. (The circles mark the boundaries of the numerical range.)

see [8, section 1.3] for a discussion of the numerical range of this A. Figure 2.1 illustrates Theorem 2.1 for $p=7$ dimensional subspaces for the Jordan block with $n=8$.

For Jordan blocks of dimension $n, \operatorname{tr}(\mathbf{H})=0$ implies that $\mu_{1}+\cdots+\mu_{n-1}=-\mu_{n}$, so for $p=n-1$,

$$
\operatorname{Re} \theta_{n-1} \geq-\frac{1}{n-1} \mu_{n}=-\frac{1}{n-1} \cos \left(\frac{\pi}{n+1}\right) \geq-\frac{1}{n-1} .
$$

The numerical range $W(\mathbf{A})$ comprises the disk of radius $\cos (\pi /(n+1))$; Theorem 2.1 establishes a containment region for the rightmost Ritz value $\theta_{n-1}$ that tends toward the right half of $W(\mathbf{A})$; see Figure 2.2. It might initially seem surprising that this bound does not require the rightmost Ritz value from a $p=n-1$ dimensional subspace to fall further to the right. However, if we take for $\mathbf{V}$ the first $n-1$ columns of the $n \times n$ identity matrix, then $\mathbf{V}^{*} \mathbf{A V}$ is the $(n-1) \times(n-1)$ upper-left corner of $\mathbf{A}$. The corresponding Ritz values are $\theta_{1}=\theta_{2}=\cdots=\theta_{n-1}=0$ : hence any bound on $\operatorname{Re} \theta_{n-1}$ must contain the interval $[0, \cos (\pi /(n+1))]$. Rightmost Ritz values with small real 
parts might be rare in practice (as indicated in the random samples in Figure 2.1), but general bounds must account for them.

To illustrate how Theorem 2.1 reveals some "inner geometry" of the numerical range, consider one more numerical experiment. We construct three nondiagonalizable matrices of dimension $n=8$ that have the same numerical range; this implies that the extreme eigenvalues of the Hermitian parts are identical. However, we pick the matrix entries so the interior eigenvalues of the Hermitian parts are quite different. We take $\mathbf{A}_{2}$ to be the $8 \times 8$ Jordan block studied previously, with ones on the superdiagonal. Then consider the matrices

$$
\mathbf{A}_{1}:=\gamma_{1}\left[\begin{array}{cccccccc}
0 & 1 & & & & & & \\
0 & 0 & & & & & & \\
& & 0 & 1 & & & & \\
& & 0 & 0 & & & & \\
& & & 0 & 1 & & \\
& & & & 0 & 0 & & \\
& & & & & 0 & 1 \\
& & & & & 0 & 0
\end{array}\right], \mathbf{A}_{3}:=\gamma_{3}\left[\begin{array}{ccccccccc}
0 & \varrho^{1} & & & & & & \\
& 0 & \varrho^{2} & & & & & \\
& & 0 & \varrho^{3} & & & & \\
& & & 0 & \varrho^{4} & & & \\
& & & & 0 & \varrho^{5} & & \\
& & & & & 0 & \varrho^{6} & \\
& & & & & & 0 & \varrho^{7} \\
& & & & & & & & 0
\end{array}\right],
$$

where unspecified entries are zero, $\varrho=1 / 8$, and $\gamma_{1}$ and $\gamma_{3}$ are chosen so $\mathbf{A}_{1}, \mathbf{A}_{2}$, and $\mathbf{A}_{3}$ have identical numerical ranges: the disk of radius $\cos (\pi / 9)$ centered at the origin.

Figure 2.3 shows computations of $p=4$ Ritz values for each of these matrices, along with the bounds from Theorem 2.1. For $\mathbf{A}_{1}$, the four containment intervals are

$\mathbf{A}_{1}$
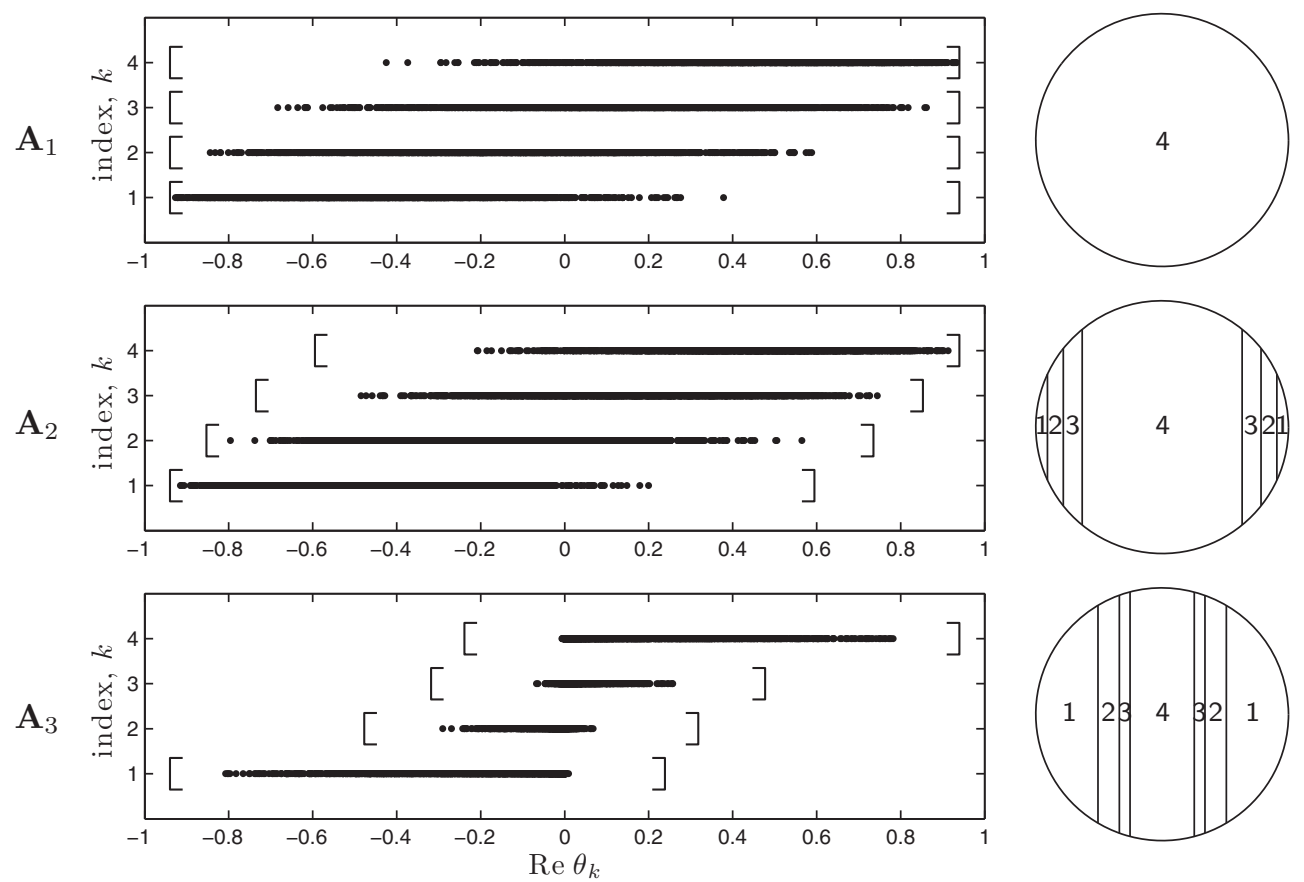

FIG. 2.3. On the left, containment intervals for the real parts of $p=4$ Ritz values for three nondiagonalizable matrices of dimension $n=8$, each with Ritz values drawn from 2000 random real p-dimensional subspaces. Each matrix has the same numerical range, shown on the right, but Theorem 2.1 reveals different "inner geometry": the numbers bound the maximum number of Ritz values that can fall in each subregion of the numerical range, from Corollary 2.2. 
the same, spanning the full breadth of the numerical range. (Indeed, due to the blockdiagonal structure of $\mathbf{A}_{1}$, these bounds are sharp.) On the other hand, the rapid decay of the superdiagonal entries in $\mathbf{A}_{3}$ causes the interior eigenvalues of $\mathbf{H}$ to be quite small, restricting the interior Ritz values from the outer extent of the numerical range.

2.3. Bounds on the magnitude of Ritz values. Classical majorization results also lead to bounds on the magnitude of Ritz values. As before, let $\mathbf{V} \in \mathbb{C}^{n \times p}$ denote a matrix with orthonormal columns, arranged so that $\mathbf{V}^{*} \mathbf{A V}$ is upper triangular with the Ritz values on the diagonal. Now label those Ritz values by decreasing magnitude, so that $\left|\theta_{1}\right| \geq \cdots \geq\left|\theta_{p}\right|$. Let the columns of $\widehat{\mathbf{V}} \in \mathbb{C}^{n \times(n-p)}$ form an orthonormal basis for $\operatorname{Ran}(\mathbf{V})^{\perp}$, chosen so that $\widehat{\mathbf{V}}^{*} \mathbf{A} \widehat{\mathbf{V}}$ is upper triangular, with eigenvalues $\theta_{p+1}, \ldots, \theta_{n}$. Relabel the values $\theta_{1}, \ldots, \theta_{n}$ by decreasing magnitude: $\left|\theta_{(1)}\right| \geq\left|\theta_{(2)}\right| \geq \cdots \geq\left|\theta_{(n)}\right|$. Let $\sigma_{j}(\cdot)$ denote the $j$ th largest singular value of a matrix. Another result of Ky Fan from 1951 majorizes the diagonal entries of a matrix by its singular values; see [14, p. 314]. Since the Ritz values are revealed along the diagonal of $[\mathbf{V} \widehat{\mathbf{V}}]^{*} \mathbf{A}[\mathbf{V} \widehat{\mathbf{V}}]$, and $[\mathbf{V} \widehat{\mathbf{V}}]$ is unitary, this gives, for $k \leq p$,

$$
k\left|\theta_{k}\right| \leq \sum_{j=1}^{k}\left|\theta_{j}\right| \leq \sum_{j=1}^{k}\left|\theta_{(j)}\right| \leq \sum_{j=1}^{k} \sigma_{j}\left([\mathbf{V} \widehat{\mathbf{V}}]^{*} \mathbf{A}[\mathbf{V} \widehat{\mathbf{V}}]\right)=\sum_{j=1}^{k} \sigma_{j}(\mathbf{A}) .
$$

Thus we have a bound on the $k$ th Ritz value. However, a better bound comes from "log-majorization": the product of the magnitudes of the $k$ largest eigenvalues of a matrix is bounded by the product of the $k$ largest singular values, a result of Weyl from 1949 [14, p. 317]. Hence for $k \leq p$,

$$
\left|\theta_{k}\right|^{k} \leq \prod_{j=1}^{k}\left|\theta_{j}\right| \leq \prod_{j=1}^{k} \sigma_{j}\left(\mathbf{V}^{*} \mathbf{A V}\right) \leq \prod_{j=1}^{k} \sigma_{j}(\mathbf{A})
$$

where the last inequality follows from the fact that $\sigma_{j}\left(\mathbf{V}^{*} \mathbf{A V}\right) \leq \sigma_{j}(\mathbf{A})$ for any matrix $\mathbf{V}$ with orthonormal columns. By the arithmetic-geometric mean inequality, the resulting inequality will never be worse than (2.4).

TheOrem 2.3. Let $\theta_{1}, \ldots, \theta_{p}$ denote the Ritz values of $\mathbf{A} \in \mathbb{C}^{n \times n}$ drawn from a $p<n$ dimensional subspace, labeled by decreasing magnitude: $\left|\theta_{1}\right| \geq \cdots \geq\left|\theta_{p}\right|$. Then for $k=1, \ldots, p$,

$$
\left|\theta_{k}\right| \leq\left(\sigma_{1}(\mathbf{A}) \cdots \sigma_{k}(\mathbf{A})\right)^{1 / k},
$$

where $\sigma_{1}(\mathbf{A}) \geq \cdots \geq \sigma_{n}(\mathbf{A})$ are the singular values of $\mathbf{A}$.

For $k=1$, this bound gives $\left|\theta_{1}\right| \leq \sigma_{1}(\mathbf{A})=\|\mathbf{A}\|$, looser than the obvious bound

$$
\left|\theta_{1}\right| \leq r(\mathbf{A}):=\max _{z \in W(\mathbf{A})}|z|,
$$

where $r(\mathbf{A})$ is called the numerical radius. It is well known that $\frac{1}{2}\|\mathbf{A}\| \leq r(\mathbf{A}) \leq\|\mathbf{A}\|$ (see, e.g., [8, p. 9]), so Theorem 2.3 can overestimate $\left|\theta_{1}\right|$ by at most a factor of two. If $\mathbf{A}$ has rank $m$, then (2.5) confirms the fact that at most $m$ Ritz values can be nonzero. (The rank of $\mathbf{V}^{*} \mathbf{A V}$ cannot exceed that of $\mathbf{A}$.)

How do these bounds perform for the Jordan blocks studied in section 2.2? Suppose $1 \leq p<n=8$. The matrix $\mathbf{A}_{1}$ has four singular values equal to $\gamma_{1}$ and all others equal to zero (it has rank 4). Hence (2.5) implies that $\left|\theta_{1}\right|, \ldots,\left|\theta_{4}\right| \leq \gamma_{1}$, while $\left|\theta_{k}\right|=0$ for $4<k \leq p$. Since $\mathbf{A}_{2}$ has seven singular values equal to one, (2.5) gives 

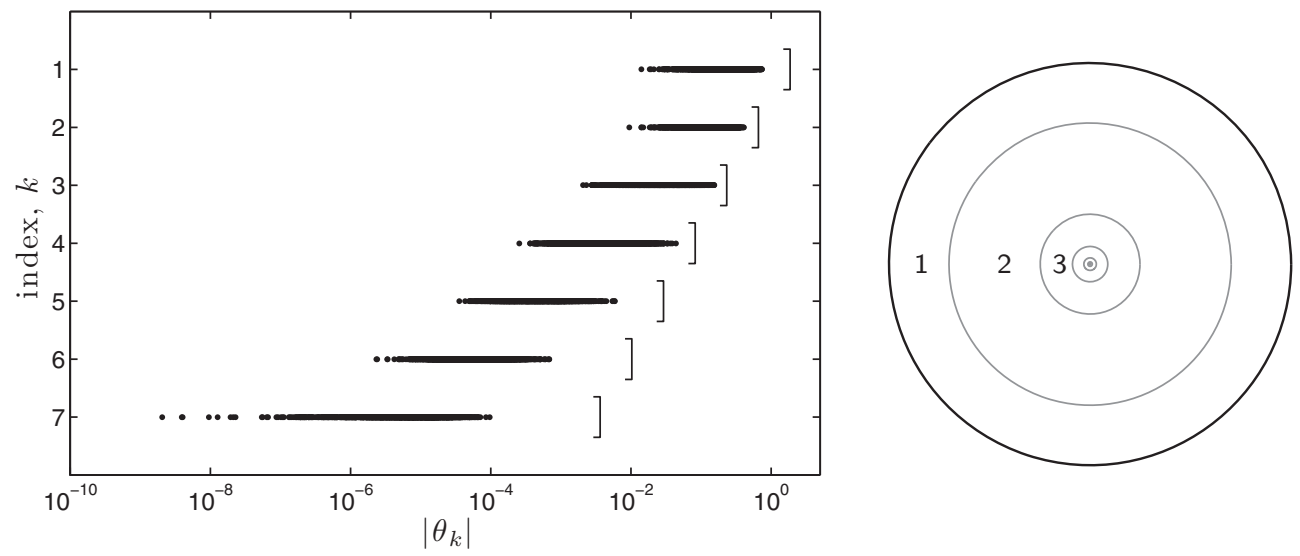

Fig. 2.4. Illustration of Theorem 2.3 for the nondiagonalizable matrix $\mathbf{A}_{3}$ from section 2.2 with $\varrho=1 / 8, n=8$, and $p=7$. The left plot shows the upper bounds on $\left|\theta_{k}\right|$, along with Ritz values from 2000 trials from random real p-dimensional subspaces. The right plot shows the numerical range $W\left(\mathbf{A}_{3}\right)$ (black circle), together with the bound (2.5) for $k=2,3, \ldots, p$ (gray circles). The numbers bound the maximum number of Ritz values that can fall between consecutive circles; e.g., at most one Ritz value can fall between the black circle and the outermost gray circle, the $k=2$ bound. (The interior circles are too close together to show such numbers.)

$\left|\theta_{1}\right|, \ldots\left|\theta_{p}\right| \leq 1$, a weaker result than given by Theorem 2.1, as seen in Figure 2.1. The matrix $\mathbf{A}_{3}$ is rather more interesting. The rapid decay of the superdiagonal entries is reflected in the singular values, so Theorem 2.3 significantly restricts the number of Ritz values that can fall near the boundary of the numerical range; see Figure 2.4. While the examples considered here are contrived, these bounds can apply to situations that arise in practical eigenvalue computations involving "shift-invert" transformations that replace $\mathbf{A}$ with $(\mathbf{A}-s \mathbf{I})^{-1}$, mapping a targeted eigenvalue $\lambda$ near $s \in \mathbb{C}$ to the largest magnitude eigenvalues $1 /(\lambda-s)$ of the transformed problem.

3. Two Ritz values of a three-dimensional Jordan block. Having developed bounds on the real parts and magnitudes of Ritz values of general matrices, we shall now examine one simple case in greater detail, illustrating that while Ritz values of nonnormal matrices can be localized, their fine behavior can be rather complicated. In particular, we shall derive expressions for the $p=2$ Ritz values of the $3 \times 3$ Jordan block (1.1) studied in the introduction with the goal of obtaining a deep understanding of the "inner geometry" of the numerical range. The size of the matrix permits a detailed analysis that gives considerable insight into the iFOV $(2)$ problem, i.e., those pairs $\left\{\theta_{1}, \theta_{2}\right\}$ of Ritz values that can be drawn from a $p=2$ dimensional subspace. Our approach is partly enabled by the perspective of algebraic geometry; starting from a parametric representation of all possible two-dimensional subspaces, we construct implicit expressions for the Ritz values. Our discussion will rigorously establish the following facts and eventually lead to numerical calculations for the boundary of the region that contains the leftmost Ritz values observed in Figure 1.1.

Proposition 3.1. Let $\mathbf{A}$ denote the $3 \times 3$ Jordan block (1.1), and suppose $\theta_{1}, \theta_{2} \in$ $W(\mathbf{A})$. Define $d:=\theta_{1} \theta_{2}, t=\theta_{1}+\theta_{2}$, and $\psi:=\arg \left(d \bar{t}^{2}\right)$. If $d \neq 0$, then $\theta_{1}, \theta_{2}$ form a valid pair of Ritz values of $\mathbf{A}$ from a two-dimensional subspace if and only if the cubic equation

$$
4|d|^{2} x^{3}+\left(12|d|^{2}-2|d|\right) x^{2}+\left(|t|^{2}+8|d|^{2}-4|d|\right) x+|t|^{2}(1-\cos \psi)=0
$$


has a root $x \in[0,(|d|+1 /|d|) / 2-1]$. If $d=0$, then $\theta_{1}$ and $\theta_{2}$ form a valid Ritz pair if and only if $|t| \leq 1 / 2$.

In particular, for any $\theta_{1} \in W(\mathbf{A}), \theta_{2}=-\theta_{1}$ is a valid Ritz value.

This detailed understanding requires an expression for the Ritz values for all possible two-dimensional subspaces. Since $p=n-1=2$, the parameterization of all subspaces is simplified by the fact that every $(n-1)$-dimensional subspace of $\mathbb{C}^{n}$, represented by $\mathbf{V} \in \mathbb{C}^{n \times(n-1)}, \mathbf{V}^{*} \mathbf{V}=\mathbf{I}$, can be characterized by any nonzero vector $\mathbf{v}$ orthogonal to the subspace, $\mathbf{V}^{*} \mathbf{v}=\mathbf{0}$. This $\mathbf{v}$, which we shall always take to be a unit vector, uniquely determines the range of $\mathbf{V}$. Any orthonormal basis for $\operatorname{Ran}(\mathbf{V})$ gives the same Ritz values. We use these facts via the matrix adjugate, as done for normal matrices in [5]. The adjugate (or classical adjoint) [9, p. 21] of a matrix satisfies

$$
[\operatorname{adj}(\mathbf{A})]_{i j}=(-1)^{i+j} \operatorname{det}\left((\mathbf{A})_{j i}\right)=(\operatorname{det} \mathbf{A})\left[\mathbf{A}^{-1}\right]_{i j},
$$

where $[\cdot]_{i j}$ refers to the $(i, j)$ element of a matrix, and $(\cdot)_{j i}$ is the matrix formed by deleting row $j$ and column $i$ from a matrix. The second equality holds only for invertible A. For unitary $\mathbf{U}$, the adjugate satisfies $\operatorname{adj}\left(\mathbf{U}^{*} \mathbf{A} \mathbf{U}\right)=\mathbf{U}^{*} \operatorname{adj}(\mathbf{A}) \mathbf{U}$. The matrix $\mathbf{U}=[\mathbf{V} \mathbf{v}] \in \mathbb{C}^{n \times n}$ is unitary, so

$$
\operatorname{det}\left(\mathbf{V}^{*} \mathbf{A V}\right)=\operatorname{det}\left(\left(\mathbf{U}^{*} \mathbf{A} \mathbf{U}\right)_{n n}\right)=\left[\operatorname{adj}\left(\mathbf{U}^{*} \mathbf{A} \mathbf{U}\right)\right]_{n n}=\left[\mathbf{U}^{*} \operatorname{adj}(\mathbf{A}) \mathbf{U}\right]_{n n}=\mathbf{v}^{*} \operatorname{adj}(\mathbf{A}) \mathbf{v}
$$

Similarly, $\operatorname{det}\left(\lambda \mathbf{I}-\mathbf{V}^{*} \mathbf{A V}\right)$, the characteristic polynomial of the restriction of a matrix A to the subspace orthogonal to $\mathbf{v}$, can be determined by computing the Rayleigh quotient of $\operatorname{adj}(\lambda \mathbf{I}-\mathbf{A})$ with $\mathbf{v}$.

When $\mathbf{A}$ is an $n \times n$ Jordan block,

$$
\operatorname{adj}(\lambda \mathbf{I}-\mathbf{A})=\sum_{j=0}^{n-1} \lambda^{n-1-j} \mathbf{A}^{j},
$$

so the coefficient of $\lambda^{j}$ in the characteristic polynomial $\operatorname{det}\left(\lambda \mathbf{I}-\mathbf{V}^{*} \mathbf{A V}\right)$ is simply $c_{j}=\mathbf{v}^{*} \mathbf{A}^{n-1-j} \mathbf{v}$. These coefficients are symmetric polynomials in the eigenvalues of $\mathbf{V}^{*} \mathbf{A V}$. For $n=3$,

$$
\begin{aligned}
\mathbf{v}^{*} \mathbf{A} \mathbf{v} & =-\left(\theta_{1}+\theta_{2}\right)=-\operatorname{tr}\left(\mathbf{V}^{*} \mathbf{A} \mathbf{V}\right) \\
\mathbf{v}^{*} \mathbf{A}^{2} \mathbf{v} & =\theta_{1} \theta_{2}=\operatorname{det}\left(\mathbf{V}^{*} \mathbf{A V}\right)
\end{aligned}
$$

where $\theta_{1}$ and $\theta_{2}$ are the eigenvalues of $\mathbf{V}^{*} \mathbf{A V}$. Without loss of generality (since $e^{i \phi} \mathbf{v}$ generates the same Ritz values for any $\phi$ ), write the unit vector $\mathbf{v}$ as

$$
\mathbf{v}=\left[\begin{array}{c}
\cos \phi_{1} \\
-\sin \phi_{1} \cos \phi_{2} e^{i \phi_{3}} \\
\sin \phi_{1} \sin \phi_{2} e^{i \phi_{4}}
\end{array}\right]
$$

for independent real parameters $\phi_{1}, \phi_{2}, \phi_{3}, \phi_{4} \in[0,2 \pi)$, thus giving

$$
\begin{aligned}
\theta_{1}+\theta_{2} & =\cos \phi_{1} \sin \phi_{1} \cos \phi_{2} e^{i \phi_{3}}+\sin ^{2} \phi_{1} \cos \phi_{2} \sin \phi_{2} e^{i\left(\phi_{4}-\phi_{3}\right)}, \\
\theta_{1} \theta_{2} & =\cos \phi_{1} \sin \phi_{1} \sin \phi_{2} e^{i \phi_{4}} .
\end{aligned}
$$

The Ritz values are completely determined by these two formulas. Hence, for there to be a two-dimensional subspace $\mathcal{V}$ that gives both $\theta_{1}$ and $\theta_{2}$ as Ritz values, there must 
exist real $\phi_{1}, \ldots, \phi_{4}$ that satisfy (3.4)-(3.5). Without loss of generality, let $\arg \theta_{1} \theta_{2}=$ $\phi_{4}$. (If $\arg \left(\theta_{1} \theta_{2}\right) \neq \phi_{4}$, one can modify $\phi_{3}$ and either $\phi_{1}$ or $\phi_{2}$ : set $\phi_{4} \rightarrow \arg \theta_{1} \theta_{2}$, $\phi_{3} \rightarrow \phi_{3}+\pi$, and either $\phi_{1} \rightarrow \pi-\phi_{1}$ or $\phi_{2} \rightarrow \phi_{2}+\pi$.) Given this parametric representation of the possible Ritz values, we seek implicit expressions relating $\theta_{1}$ and $\theta_{2}$. From these expressions, we will find the number of distinct subspaces that generate a given pair of Ritz value combinations and, where possible, give formulas for $\mathbf{v}$ in terms of $\theta_{1}$ and $\theta_{2}$.

3.1. A Ritz value at zero. We wish to use (3.5) to eliminate $\phi_{4}$ from (3.4). To perform this elimination, $\cos \phi_{1} \sin \phi_{1} \sin \phi_{2}$ must be nonzero. First we address the special case where $\cos \phi_{1} \sin \phi_{1} \sin \phi_{2}=0$, which implies by (3.5) that at least one of the Ritz values is zero; say, $\theta_{1}=0$. Three scenarios are possible from (3.4):

- $\sin \phi_{1}=0$, in which case $\theta_{2}=0$;

- $\cos \phi_{1}=0$, in which case $\theta_{2}=\cos \phi_{2} \sin \phi_{2} e^{i\left(\phi_{4}-\phi_{3}\right)}$, allowing $\theta_{2}$ to take any value in the disk $\{z \in \mathbb{C}:|z| \leq 1 / 2\}$;

- $\sin \phi_{2}=0$, in which case $\theta_{2}= \pm \cos \phi_{1} \sin \phi_{1} e^{i \phi_{3}}$, allowing $\theta_{2}$ to take any value in the disk $\{z \in \mathbb{C}:|z| \leq 1 / 2\}$.

Hence, any pair of Ritz values $\{0, \theta\}$ is possible for $|\theta| \leq 1 / 2$ : $\theta=0$ only corresponds to the subspaces defined by $\mathbf{v} \in\left\{\mathbf{e}_{1}, \mathbf{e}_{2}, \mathbf{e}_{3}\right\}$, the set of canonical basis vectors; each $0<|\theta|<1 / 2$ corresponds to the four subspaces orthogonal to one of the vectors

$$
\mathbf{v}=\left[\begin{array}{c}
0 \\
-\sqrt{\frac{1 \pm \sqrt{1-4|\theta|^{2}}}{2}} \\
\frac{\theta}{|\theta|} \sqrt{\frac{1 \mp \sqrt{1-4|\theta|^{2}}}{2}}
\end{array}\right], \quad \mathbf{v}=\left[\begin{array}{c}
-\sqrt{\frac{1 \mp \sqrt{1-4|\theta|^{2}}}{2}} \\
\frac{\theta}{|\theta|} \sqrt{\frac{1 \pm \sqrt{1-4|\theta|^{2}}}{2}} \\
0
\end{array}\right]
$$

For these $\mathbf{v}$, the subspace $\operatorname{Ran}(\mathbf{V})=\mathbf{v}^{\perp}$ must contain either a left or a right eigenvector of $\mathbf{A}$. For $|\theta|=1 / 2$ there are only two choices of $\mathbf{v} .^{2}$ Already, we see that if one Ritz value is at zero, the other cannot be near the boundary of $W(\mathbf{A})$, i.e., in the region $\{z \in \mathbb{C}: 1 / 2<|z| \leq \sqrt{2} / 2\}$. This set is shown, along with similar regions for fixed nonzero Ritz values, in Figure 3.2 at the end of this section.

3.2. Zero trace. Having handled all $\theta_{1} \theta_{2}=0$ cases, now assume $\theta_{1} \theta_{2} \neq 0$. Using (3.5), substitute

$$
e^{i \phi_{4}}=\frac{\theta_{1} \theta_{2}}{\cos \phi_{1} \sin \phi_{1} \sin \phi_{2}}
$$

into (3.4) to eliminate $\phi_{4}$ :

$$
\left(\cos ^{2} \phi_{1} \sin \phi_{1} e^{i \phi_{3}}+\theta_{1} \theta_{2} \sin \phi_{1} e^{-i \phi_{3}}\right) \cos \phi_{2}=\left(\theta_{1}+\theta_{2}\right) \cos \phi_{1} .
$$

If the expression on the left is zero, so too must be the expression on the right. Thus $\theta_{1}+\theta_{2}=0$, since $\cos \phi_{1}=0$ gives $\theta_{1} \theta_{2}=0$, handled above. If the coefficient of $\cos \phi_{2}$ on the left of (3.7) is zero, then $\theta_{1}^{2}=\cos ^{2} \phi_{1} e^{2 i \phi_{3}}$; if $\cos \phi_{2}=0$, then $-\theta_{1}^{2}=$ $\cos \phi_{1} \sin \phi_{1} e^{i \phi_{4}}$. Together, these cases give four possible solutions, corresponding to

\footnotetext{
${ }^{2}$ For this nonnormal $\mathbf{A}$, the entries of $\mathbf{v}$ are uniquely determined by functions that involve square roots of the Ritz values; in contrast, for normal matrices the analogous formulas only involve polynomial expressions of $\theta_{1}$ and $\theta_{2}$ [5].
} 
the vectors

$$
\mathbf{v}=\left[\begin{array}{c}
\overline{\theta_{1}} \\
\pm \sqrt{1-2\left|\theta_{1}\right|^{2}} \\
-\theta_{1}
\end{array}\right], \quad \mathbf{v}=\left[\begin{array}{c}
\frac{\overline{\theta_{1}}}{\left|\theta_{1}\right|} \sqrt{\frac{1 \mp \sqrt{1-4\left|\theta_{1}\right|^{4}}}{2}} \\
0 \\
-\frac{\theta_{1}}{\left|\theta_{1}\right|} \sqrt{\frac{1 \pm \sqrt{1-4\left|\theta_{1}\right|^{4}}}{2}}
\end{array}\right] .
$$

For $\theta_{1}=\sqrt{2} / 2$ on the boundary of $W(\mathbf{A})$, there is one vector: $\mathbf{v}=[\sqrt{2} / 2,0,-\sqrt{2} / 2]^{\mathrm{T}}$.

These calculations imply that any pair $\theta_{1}, \theta_{2} \in W(\mathbf{A})$ satisfying $\theta_{1}=-\theta_{2}$ is a valid Ritz pair from some two-dimensional subspace.

3.3. Some conjugate pairs. Continuing with a nonzero determinant, $\theta_{1} \theta_{2} \neq 0$, we can now assume the coefficient of $\cos \phi_{2}$ on the left of (3.7) is nonzero. Then from (3.7),

$$
\cos \phi_{2}=\frac{\left(\theta_{1}+\theta_{2}\right) \cos \phi_{1}}{\left(\cos ^{2} \phi_{1} e^{i \phi_{3}}+\theta_{1} \theta_{2} e^{-i \phi_{3}}\right) \sin \phi_{1}}
$$

thus determining $\phi_{2}$ in terms of $\phi_{1}, \phi_{3}$, and the Ritz values.

To simplify the coefficients, write $d:=\theta_{1} \theta_{2}$ and $t:=\theta_{1}+\theta_{2}$ for the determinant and trace of $\mathbf{V}^{*} \mathbf{A V}$, so

$$
\cos \phi_{2}=\frac{t \cos \phi_{1}}{\left(\cos ^{2} \phi_{1} e^{i \phi_{3}}+d e^{-i \phi_{3}}\right) \sin \phi_{1}} .
$$

Requiring the imaginary part of (3.9) to be zero yields

$$
\left(\operatorname{Im}(t) \cos ^{2} \phi_{1}-\operatorname{Im}(d \bar{t})\right) \cos \phi_{3}=\left(\operatorname{Re}(t) \cos ^{2} \phi_{1}-\operatorname{Re}(d \bar{t})\right) \sin \phi_{3},
$$

and so

$$
\tan \phi_{3}=\frac{\operatorname{Im}(t) \cos ^{2} \phi_{1}-\operatorname{Im}(d \bar{t})}{\operatorname{Re}(t) \cos ^{2} \phi_{1}-\operatorname{Re}(d \bar{t})}
$$

Hence $\phi_{3}$ is ill-defined when the coefficients of $\cos \phi_{3}$ and $\sin \phi_{3}$ in (3.10) are both zero, i.e., when $t \cos ^{2} \phi_{1}=d \bar{t}$. In this subsection we analyze this special situation, then return to the general case in section 3.4.

The expression $t \cos ^{2} \phi_{1}=d \bar{t}$ is invariant to rotations of the Ritz values about the origin in the complex plane, since a rotation of both Ritz values by the angle $\gamma$ corresponds to multiplying the determinant by $e^{2 i \gamma}$ and the trace by $e^{i \gamma}$ :

$$
\left(t e^{i \gamma}\right) \cos ^{2} \phi_{1}=\left(d e^{2 i \gamma}\right) \overline{t e^{i \gamma}}
$$

Hence we can assume that the trace is real and positive, and since $t \cos ^{2} \phi_{1}=d \bar{t}$, the determinant is also real and positive.

With $d=\cos ^{2} \phi_{1}$ and $t>0$, we can use $\phi_{4}=0$ and (3.5) to conclude $\cos ^{2} \phi_{2}=$ $(2 d-1) /(d-1)$. Substituting this expression into (3.9) gives

$$
8 d^{2}-4 d+t^{2} \sec ^{2} \phi_{3}=0 .
$$

One can show that the only real Ritz values consistent with (3.12) are $\theta_{1}=\theta_{2}=0$, a case covered earlier. Hence the Ritz values considered here must form complex 
conjugate pairs. Letting $\theta=x+i y$, we have $d=x^{2}+y^{2}$ and $t=2 x$, so (3.12) implies

$$
\begin{aligned}
0 & =2\left(x^{2}+y^{2}\right)^{2}-\left(x^{2}+y^{2}\right)+x^{2} \sec ^{2} \phi_{3} \\
& \geq 2\left(x^{2}+y^{2}\right)^{2}-\left(x^{2}+y^{2}\right)+x^{2} \\
& =2\left(\left(x^{2}-\frac{1}{8}\right)+\left(y-\frac{\sqrt{2}}{4}\right)^{2}\right)\left(\left(x^{2}-\frac{1}{8}\right)+\left(y+\frac{\sqrt{2}}{4}\right)^{2}\right) \\
& =2\left(\left|\theta-i \frac{\sqrt{2}}{4}\right|^{2}-\frac{1}{8}\right)\left(\left|\theta+i \frac{\sqrt{2}}{4}\right|^{2}-\frac{1}{8}\right) .
\end{aligned}
$$

This last expression is nonpositive for all $\theta$ in the union of the closed disks of radius $\sqrt{2} / 4$ centered at $\pm i \sqrt{2} / 4$ in the complex plane: all the Ritz values for this scenario $\left(d \neq 0, t>0, d=\cos ^{2} \phi_{1}\right)$ come in complex conjugate pairs and lie in these disks.

Ritz values corresponding to $d \neq 0, t \neq 0$, and $t \cos ^{2} \phi_{1}=d \bar{t}$ correspond to two possible values for $\mathbf{v}$ in the form (3.3):

$$
\mathbf{v}=\left[\begin{array}{c}
\overline{\sqrt{d}} \\
-\frac{|t|}{2 \sqrt{|d|}} \pm i \sqrt{1-2|d|-\frac{|t|^{2}}{4|d|}} \\
\sqrt{d}
\end{array}\right],
$$

where $\sqrt{d}$ is chosen such that $\arg (\sqrt{d})=\arg (t)$. If $t=0$, this expression essentially reduces to the first vector in (3.8). In the special case of $1-2|d|-|t|^{2} / 4|d|=0,(3.13)$ gives just one vector, generating Ritz values that lie on the boundary of the disks mentioned above (suitably rotated by $e^{i \gamma}$ ).

3.4. General case. Return now to (3.9). Having analyzed $t \cos ^{2} \phi_{1}=d \bar{t}$, we can address the general case, where $t \cos ^{2} \phi_{1} \neq d \bar{t}$. Equation (3.10), together with the requirement that $e^{i \phi_{3}}$ must have unit modulus, gives

$$
e^{i \phi_{3}}=\frac{t \cos ^{2} \phi_{1}-d \bar{t}}{\left|t \cos ^{2} \phi_{1}-d \bar{t}\right|} .
$$

The expressions for $\phi_{2}, \phi_{3}$, and $\phi_{4}$ in (3.9), (3.14), and (3.6) provide an expression for $\mathbf{v}$ in terms of $d, t$, and $\cos \phi_{1}$ :

$$
\mathbf{v}=\left[\begin{array}{c}
\cos \phi_{1} \\
-\frac{\cos \phi_{1}\left(t \cos ^{2} \phi_{1}-d \bar{t}\right)}{\cos ^{4} \phi_{1}-|d|^{2}} \\
\frac{d}{\cos \phi_{1}}
\end{array}\right] .
$$

The second entry has a pole at $\cos ^{2} \phi_{1}=|d|$; the residue at this pole is zero if and only if $\arg \left(d \bar{t}^{2}\right)=0$, which corresponds to Ritz values that are equivalent to a complex conjugate pair, as handled in the last subsection. For this vector to have norm one, $\cos \phi_{1}$ must satisfy

$$
\begin{aligned}
0= & \left(\cos ^{12} \phi_{1}+|d|^{6}\right)-\left(\cos ^{10} \phi_{1}+|d|^{4} \cos ^{2} \phi_{1}\right) \\
& +\left(|t|^{2}-|d|^{2}\right)\left(\cos ^{8} \phi_{1}+|d|^{2} \cos ^{4} \phi_{1}\right)+\left(2|d|^{2}-d \bar{t}^{2}-\bar{d} t^{2}\right) \cos ^{6} \phi_{1} .
\end{aligned}
$$

The right-hand side is a polynomial in $\cos \phi_{1}$ that involves only even powers, consistent with $\pm \mathbf{v}$ generating the same subspace $\operatorname{Ran}(\mathbf{V})$. The terms are arranged to emphasize 
that the polynomial is $|d|^{2}$-self-reciprocal, i.e., if $\cos ^{2} \phi_{1}$ is a solution, then $|d|^{2} / \cos ^{2} \phi_{1}$ is also a solution: a consequence of $\mathbf{A}$ being similar to its transpose via a permutation. Using the $|d|^{2}$-self-reciprocal property, make the substitution $\cos ^{2} \phi_{1} \rightarrow|d| e^{y}$ to reduce (3.16) to

$$
0=4|d|^{2} \cosh ^{3} y-2|d| \cosh ^{2} y+\left(-4|d|^{2}+|t|^{2}\right) \cosh y-|t|^{2} \cos \psi+2|d|
$$

where $\psi:=\arg \left(d \bar{t}^{2}\right)$. This equation is cubic in $\cosh y$, so one can write out the solution exactly in terms of $d, t$, and $\cos \psi$; however, the complexity of the expressions limits the amount of insight that can be gained. Numerical calculations indicate that at most two of the solutions to this equation correspond to actual Ritz value pairs. This would imply that the generic case gives at most four distinct subspaces that generate the same pair of Ritz values.

3.5. Solving iFOV(2). Having carefully studied the relationship between the Ritz values and their generating subspaces, we return to our main motivation, the iFOV (2) problem for the Jordan block: given two candidate Ritz values $\theta_{1}, \theta_{2} \in$ $W(\mathbf{A})$, determine if there exists some two-dimensional subspace such that $\theta_{1}$ and $\theta_{2}$ are simultaneously Ritz values of $\mathbf{A}$. The general analysis of the last subsection enables an easy test for solutions to $\operatorname{iFOV}(2)$.

Given $\theta_{1}$ and $\theta_{2}$, form $\psi:=\arg \left(d \bar{t}^{2}\right)$ and let $\delta:=|d|$ and $\tau:=|t|$. Since $\cosh (y) \geq 1$ for real $y$, define $x:=\cosh (y)-1$. Now expand (3.17) as a cubic polynomial in $x$ :

$$
4 \delta^{2} x^{3}+\left(12 \delta^{2}-2 \delta\right) x^{2}+\left(\tau^{2}+8 \delta^{2}-4 \delta\right) x+\tau^{2}(1-\cos \psi)=0 .
$$

We want to show that all roots $x$ that correspond to solutions of $\mathrm{iFOV}(2)$ must be in the interval $[0,(\delta+1 / \delta) / 2-1]$. As $\cos ^{2} \phi_{1}=\delta e^{y}$ and $0 \leq \cos ^{2} \phi_{1} \leq 1$, we have $e^{y} \in[0,1 / \delta]$; the $\delta^{2}$-self-reciprocal property of (3.16) ensures that $\delta^{2} / \cos ^{2} \phi_{1}=\delta e^{-y}$ is also a root, and so we must also have $e^{-y} \in[0,1 / \delta]$. These requirements together give $e^{ \pm y} \in[\delta, 1 / \delta]$, so $\cosh y \in[1,(\delta+1 / \delta) / 2]$, i.e., all valid roots $x$ must be in the interval $[0,(\delta+1 / \delta) / 2-1]$. This gives a quick way to check if $\mathrm{iFOV}(2)$ is solvable.

- Given $\theta_{1}, \theta_{2} \in W(\mathbf{A})$, form $\psi:=\arg \left(d \bar{t}^{2}\right), \delta:=\left|\theta_{1} \theta_{2}\right|$, and $\tau:=\left|\theta_{1}+\theta_{2}\right|$.

- Compute the roots of the cubic equation (3.18).

- If at least one root $x \in[0,(\delta+1 / \delta) / 2-1]$, then iFOV $(2)$ has a valid solution.

This test enables one to check the solvability of $\mathrm{iFOV}(2)$ numerically for a candidate pair of Ritz values. However, considerable additional structure about the $(\delta, \tau)$ pairs for which $\mathrm{iFOV}(2)$ is solvable can be discovered if one is willing to dig deeper into (3.18). Descartes' rule of signs (see, e.g., [18, p. 319]) characterizes when (3.18) can have real positive roots by counting the sign changes in the ordered nonzero (real-valued) coefficients. The first coefficient $4 \delta^{2}$ is positive for $\delta>0$. The second coefficient $12 \delta^{2}-2 \delta$ is negative for $\delta \in(0,1 / 6)$ and positive for $\delta>1 / 6$. The third coefficient $\tau^{2}+8 \delta^{2}-4 \delta$ is negative in the interior of an ellipse in the $(\delta, \tau)$ plane centered at $(1 / 4,0)$ with semimajor and semiminor axes of length $\sqrt{2} / 2$ and $1 / 4$ and positive on the exterior of this ellipse. The last coefficient is always nonnegative, being zero when $\cos \psi=1$. We subdivide the relevant part of the $(\delta, \tau)$ plane into four regions depending on the signs of the middle two coefficients; see Figure 3.1.

\begin{tabular}{r|c|c|c|c}
\multicolumn{1}{c}{$12 \delta^{2}-2 \delta$} & $\tau^{2}+8 \delta^{2}-4 \delta$ & $\#$ sign changes & \# positive roots \\
\cline { 2 - 5 } I & - & - & 2 & 0 or 2 \\
\cline { 2 - 5 } II & - & + & 2 & 0 or 2 \\
\cline { 2 - 5 } III & + & - & 2 & 0 or 2 \\
\cline { 2 - 5 } IV & + & + & 0 & 0
\end{tabular}

Copyright $@$ ㅇ by SIAM. Unauthorized reproduction of this article is prohibited. 

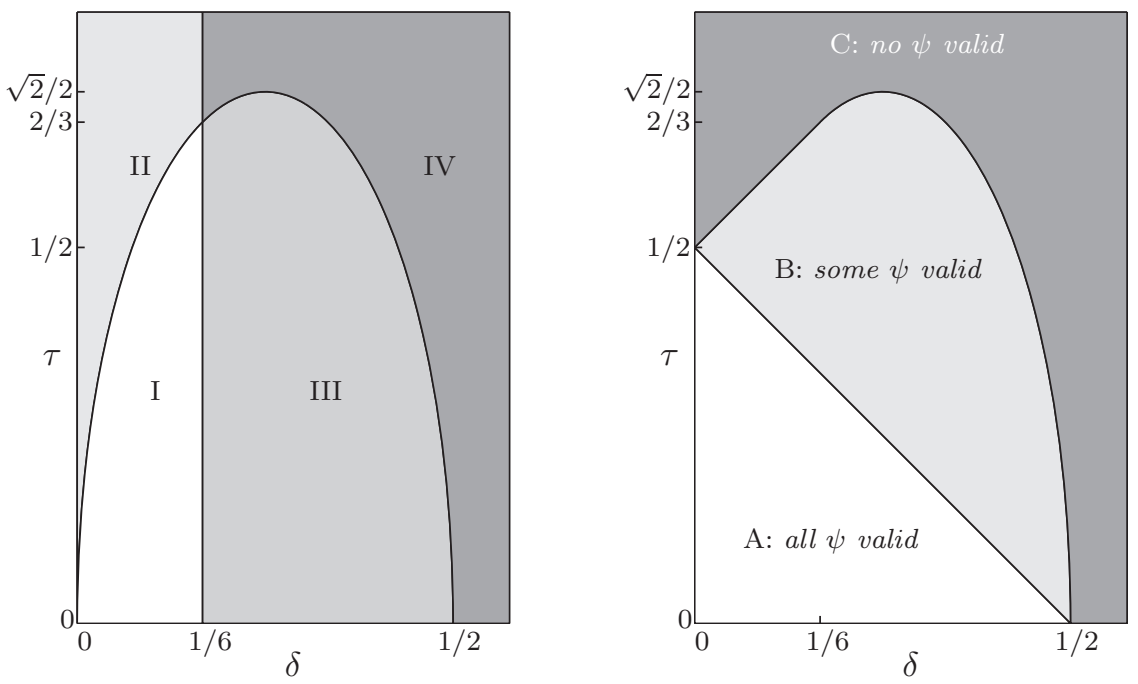

FIG. 3.1. Regions I, II, III, and IV (left), and A, B, and C (right) mapping $\delta=|d|=\left|\theta_{1} \theta_{2}\right|$ and $\tau=|t|=\left|\theta_{1}+\theta_{2}\right|$ to valid pairs of $\theta_{1}$ and $\theta_{2}$ via $\psi=\arg \left(d \bar{t}^{2}\right)$.

For regions I, II, and III we must check that there are two positive roots, then verify that they fall in the interval $[0,(\delta+1 / \delta) / 2-1]$. Applying Descartes' rule of signs to (3.18) about the point $x=(\delta+1 / \delta) / 2-1$ confirms that all real positive roots of $(3.18)$ indeed fall in the interval $[0,(\delta+1 / \delta) / 2-1]$ for all $(\delta, \tau) \in \mathrm{I} \cup \mathrm{II} \cup \mathrm{III}$.

From (3.1) and (3.2), we already know that valid $(\delta, \tau)$ combinations must lie in $[0,1 / 2] \times[0, \sqrt{2} / 2]$. (Note that $\tau \leq \sqrt{2} / 2$ from the argument at the end of the introduction.) Thus all valid $(\delta, \tau)$ combinations are in the union of the rectangle $[0,1 / 6] \times[0, \sqrt{2} / 2]$ and the elliptical region $\tau^{2}+8 \delta^{2}-4 \delta \leq 0$ in the positive orthant; see Figure 3.1. Before refining the region of valid $(\delta, \tau)$ combinations, we note the locations in the $(\delta, \tau)$ plane of the limiting cases from sections 3.1, 3.2, and 3.3.

In general, roots of (3.18) correspond to two solutions for $\mathrm{iFOV}(2)$, i.e., two distinct two-dimensional subspaces that give $\theta_{1}$ and $\theta_{2}$ as Ritz values (again a consequence of the similarity of $\mathbf{A}$ to its transpose via a permutation). Section 3.1 analyzed $\delta=0$, i.e., points on the left of both plots in Figure 3.1. Here we have three solutions of $\operatorname{iFOV}(2)$ for $\tau=0$, four solutions for $\tau \in(0,1 / 2)$, two solutions for $\tau=1 / 2$, and no solutions for $\tau>1 / 2$.

Section 3.2 addressed $\tau=0$, the bottom of the plots in Figure 3.1. For $\delta \in(0,1 / 2]$ we have four solutions of $\operatorname{iFOV}(2)$, and for $\delta=1 / 2$ we have only one solution.

In section $3.3,8 \delta^{2}-4 \delta+\tau^{2} \leq 0$ and $\cos \psi=1$; the first criterion gives $(\delta, \tau) \in$ I $\cup$ III. (Now we must consider the value of $\cos \psi$, whereas in the previous cases $\cos \psi:=\arg \left(d \bar{t}^{2}\right)$ was ill-defined, since either $\delta$ or $\tau$ was zero.) Inside the ellipse and for $\cos \psi=1$ we have at least two solutions of $\operatorname{iFOV}(2)$, while on the boundary of the ellipse we have at least one solution.

Now we can handle the general case.

1. We need (3.18) to have at least one solution $x \in[0,(\delta+1 / \delta) / 2-1]$.

2. Descartes' rule of signs on (3.18) divides the $(\delta, \tau)$ plane into four regions: region IV can be immediately discarded; we must test if the other regions support viable solutions for some $\psi$ values.

3. Descartes' rule of signs on (3.18) with appropriate substitutions for $x$ gives the following for all $(\delta, \tau) \in \mathrm{I} \cup \mathrm{II} \cup \mathrm{III}$ : 
(a) (3.18) always has exactly one negative root;

(b) all nonnegative roots of (3.18) fall in the interval $[0,(\delta+1 / \delta) / 2-1]$.

4. Consider the discriminant [11] of the cubic (3.18) with real-valued coefficients:

$$
\begin{aligned}
& 4 \delta^{2}\left(16 \delta^{2}-128 \delta^{4}+256 \delta^{6}-80 \delta^{2} \tau^{2}-192 \delta^{4} \tau^{2}+\tau^{4}+48 \delta^{2} \tau^{4}\right. \\
& \left.\quad-4 \tau^{6}-8 \delta \tau^{2} \cos \psi+288 \delta^{3} \tau^{2} \cos \psi+36 \delta \tau^{4} \cos \psi-108 \delta^{2} \tau^{4} \cos ^{2} \psi\right)
\end{aligned}
$$

(a) If this discriminant is negative, then (3.18) has one real root: Descartes' rule of signs already showed there must be either zero or two positive roots, so in this case (3.18) has no positive roots.

(b) If the discriminant is zero, all roots are real, with one of them a double root. Given the above observations, this double root must be nonnegative.

(c) If the discriminant is positive, all roots are real and distinct, so there must be two nonnegative solutions.

5. Now ascertain the sign of the discriminant, seeking regions where we can make a definitive statement about the solvability of $\operatorname{iFOV}(2)$ for all $\psi$ or for some subset of $\psi$.

(a) The discriminant is quadratic in $\cos \psi$, with negative leading coefficient; hence it opens down. If it has real roots, then for all $\cos \psi$ values between the roots, the discriminant is positive. With the aid of rather technical symbolic calculations, we can identify three regions of the $(\delta, \tau)$ plane, illustrated in the right plot in Figure 3.1:

A: For $0<\delta<1 / 2$ and $0<\tau<1 / 2-\delta$, the Ritz value pair exists for all $\cos \psi \in[-1,1]$; hence any value of $\psi:=\arg \left(d \bar{t}^{2}\right)$ is valid.

B: For $0<\delta<1 / 6$ and $1 / 2-\delta<\tau<1 / 2+\delta$, or $1 / 6<\delta<1 / 2$ and $1 / 2-\delta<\tau<\sqrt{4 \delta-8 \delta^{2}}$, the Ritz value pair exists only for some $\cos \psi \in[-1,1]$; some values of $\cos \psi \in[-1,1]$ do not correspond to valid Ritz value pairs.

C: For all other values of $\delta$ and $\tau$, no choice of $\cos \psi \in[-1,1]$ will yield solutions; such $\delta$ and $\tau$ never correspond to valid Ritz value pairs.

In summary, if $\theta_{1}$ and $\theta_{2}$ correspond to $\delta=\left|\theta_{1} \theta_{2}\right|$ and $\tau=\left|\theta_{1}+\theta_{2}\right|$ that fall in region $\mathrm{A}, \mathrm{iFOV}(2)$ is solvable; in region $\mathrm{B}, \mathrm{iFOV}(2)$ might be solvable, depending on $\psi=\arg \left(\theta_{1} \theta_{2}{\overline{\left(\theta_{1}+\theta_{2}\right)}}^{2}\right)$; in region $\mathrm{C}$, $\mathrm{iFOV}(2)$ is not solvable.

3.6. Restricting leftmost Ritz values. Given $\theta_{1}$, the results of the previous sections do not immediately reveal where $\theta_{2}$ can be located; however, they do suggest a recipe for determining such regions. Without loss of generality we may assume that $\theta_{1}$ is real and nonnegative. From the definition of trace and determinant, the following equations relate $\tau, \delta$, and $\cos \psi$ to $\theta_{1}$ and $\theta_{2}$ :

$$
\begin{aligned}
\delta^{2} & =\theta_{1}^{2}\left(\left(\operatorname{Re} \theta_{2}\right)^{2}+\left(\operatorname{Im} \theta_{2}\right)^{2}\right), \\
\tau^{2} & =\left(\theta_{1}+\operatorname{Re} \theta_{2}\right)^{2}+\left(\operatorname{Im} \theta_{2}\right)^{2}, \\
\cos \psi & =\frac{\theta_{1} \operatorname{Re}\left(\theta_{2}\left(\theta_{1}+\overline{\theta_{2}}\right)^{2}\right)}{\delta \tau^{2}} .
\end{aligned}
$$

For fixed $\theta_{1} \geq 0$ and $\operatorname{Re} \theta_{2}$ we can see that as $\operatorname{Im} \theta_{2}$ increases, so do $\delta$ and $\tau$. From 

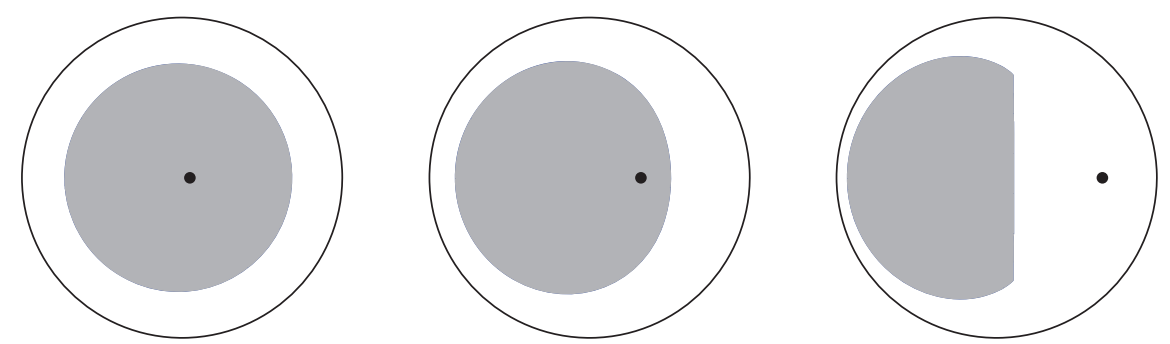

Fig. 3.2. For $\mathbf{A}$ equal to the three-dimensional Jordan block, the outermost circle shows the boundary of $W(\mathbf{A})$. For three fixed choices of $\theta_{1}(\bullet)$, the gray regions show where $\theta_{2}$ can fall.

these equations we can determine a hyperbola in the $(\delta, \tau)$ plane,

$$
\tau^{2}-\delta^{2} / \theta_{1}^{2}=\theta_{1}^{2}+2 \theta_{1} \operatorname{Re} \theta_{2}
$$

The hyperbola opens up for $\operatorname{Re} \theta_{2}>-\theta_{1} / 2$ and to the right otherwise. For this fixed $\theta_{1}$ and $\operatorname{Re} \theta_{2}$, we seek all $\operatorname{Im} \theta_{2}$ values for which $\theta_{1}$ and $\theta_{2}$ can simultaneously be Ritz values. For a point $(\delta, \tau)$ on the hyperbola (3.21) to correspond to a valid Ritz pair, we must be able to find a real value for $\operatorname{Im} \theta_{2}$ from the given $\theta_{1}$ and $\operatorname{Re} \theta_{2}$ via the equations $\delta=\left|\theta_{1} \theta_{2}\right|$ and $\tau=\left|\theta_{1}+\theta_{2}\right|$. The leftmost point in the first quadrant of the $(\delta, \tau)$ plane along the hyperbola (3.21) that gives a real value for $\operatorname{Im} \theta_{2}$ occurs when $\operatorname{Im} \theta_{2}=0$, i.e., $(\delta, \tau)=\left(\theta_{1}\left|\operatorname{Re} \theta_{2}\right|,\left|\theta_{1}+\operatorname{Re} \theta_{2}\right|\right)$. The range of $\operatorname{Im} \theta_{2}$ for which $\mathrm{iFOV}(2)$ is solvable corresponds to when the curve (3.20) is inside the region of feasible $(\delta, \tau, \cos \psi)$. From symmetry, we know that the set of permissible $\operatorname{Im} \theta_{2}$ is symmetric about the real axis. We have numerically observed that this set does not have any gaps, i.e., if iFOV(2) is solvable for given $\theta_{1} \geq 0$ and $\operatorname{Re} \theta_{2}$, then $\operatorname{Im} \theta_{2}$ can lie anywhere in some interval $[-\alpha, \alpha]$. In Figure 3.2, we show the regions where $\theta_{2}$ must lie in order for $\operatorname{iFOV}(2)$ to be solvable for three different values of $\theta_{1}$.

Finally, we return to the plot that began this investigation, Figure 1.1. Can we calculate a sharp boundary for the region that contains the leftmost Ritz value?

Let $\Theta_{L}$ denote the set of all leftmost Ritz values from two-dimensional subspaces, i.e., the set of all $\theta_{1} \in W(\mathbf{A})$ such that there exists some valid corresponding Ritz value $\theta_{2}$ with $\operatorname{Re}\left(\theta_{1}\right) \leq \operatorname{Re}\left(\theta_{2}\right)$.

We wish to characterize the boundary of $\Theta_{L}$. From the majorization bounds in section 2, the real part of any point on the boundary of $\Theta_{L}$ must be less than or equal to $\sqrt{2} / 4$; this gives the bound in Figure 1.1. We claim that for any real, positive $\theta_{1}$, if $\theta_{1}>\operatorname{Re} \theta_{2}$ for all valid $\theta_{2}$, then there exists a unique $\phi_{*} \in[0, \pi / 2]$ such that $\operatorname{Re}\left(\theta_{1} e^{i \phi_{*}}\right) \geq \operatorname{Re}\left(\theta_{2} e^{i \phi_{*}}\right)$ for all valid $\theta_{2}$, with equality for at least one $\theta_{2}$; see the left plot in Figure 3.3. First, such a $\phi_{*}$ must be less than or equal to $\pi / 2$, since there always exists a restriction such that $\theta_{2}=-\theta_{1}$ (as in section 3.2). Second, equality must be attained for some $\theta_{2}$, as the set of $\theta_{2}$ for a given $\theta_{1}$ is determined by the intersection of two closed sets in $(\delta, \tau, \cos \psi)$ space, and by the previous remark we know this intersection is not empty. Last, the uniqueness of $\phi_{*}$ follows from the attainment of equality in $\operatorname{Re}\left(\theta_{1} e^{i \phi_{*}}\right) \geq \operatorname{Re}\left(\theta_{2} e^{i \phi_{*}}\right)$ for some $\theta_{2}$, since for any $\phi \in\left(\phi_{*}, \pi / 2\right]$ there must exist at least one $\theta_{2}$ such that $\operatorname{Re}\left(\theta_{1} e^{i \phi}\right) \leq \operatorname{Re}\left(\theta_{2} e^{i \phi}\right)$. Thus $\theta_{1} e^{i \phi_{*}}$ must be on the boundary separating $\Theta_{L}$ from $\Theta_{L} \backslash W(\mathbf{A})$, as we also have $\operatorname{Re}\left(\theta_{1} e^{i \phi}\right)>\operatorname{Re}\left(\theta_{2} e^{i \phi}\right)$ for $\phi \in\left[0, \phi_{*}\right)$ and for all valid $\theta_{2}$. (If $\left\{\theta_{1}, \theta_{2}\right\}$ is a valid Ritz pair, so too is $\left\{\theta_{1} e^{i \phi}, \theta_{2} e^{i \phi}\right\}$.) The boundaries of $W(\mathbf{A})$ and $\Theta_{L}$ coincide in the left half-plane, which follows from the same argument with $\theta_{1}=\sqrt{2} / 2$. Figure 3.3 

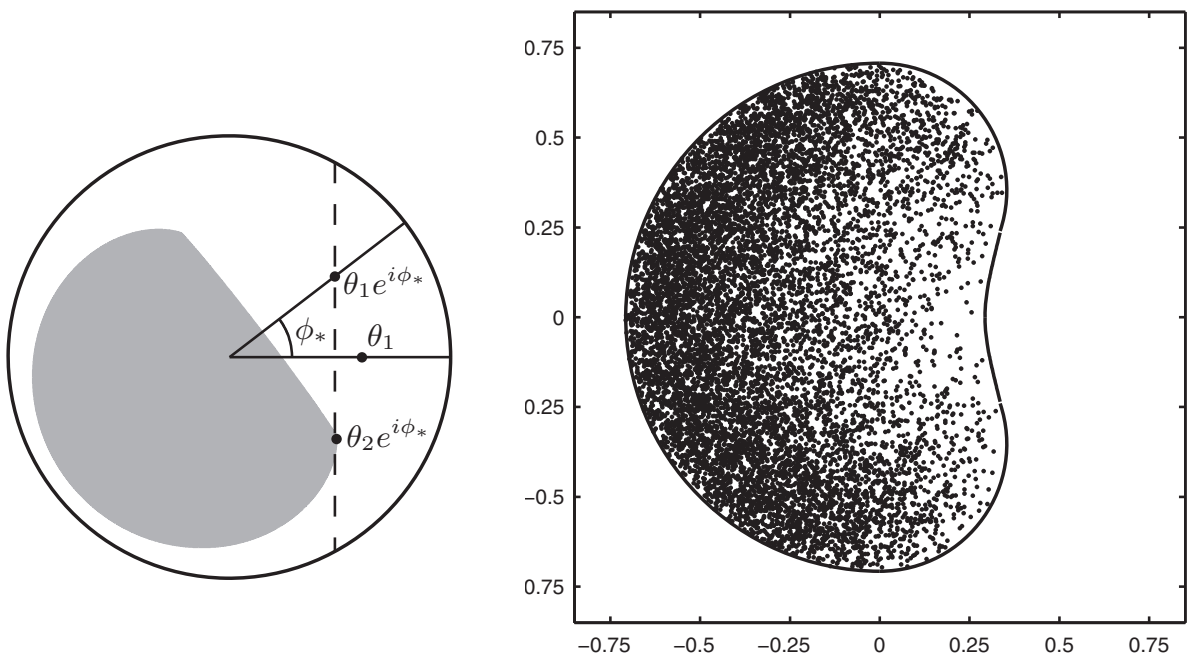

FIG. 3.3. Determination of the region $\Theta_{L}$ comprising all leftmost Ritz values from pairs $\left\{\theta_{1}, \theta_{2}\right\}$ drawn from two-dimensional subspaces of the $3 \times 3$ Jordan block. Left plot: From a prescribed real positive value $\theta_{1}$, a point on the boundary can be computed from knowledge of all valid corresponding $\theta_{2}$, as described in the text. Here the gray region shows all Ritz values $\theta_{2} e^{i \phi_{*}}$ corresponding to the fixed Ritz value $\theta_{1} e^{i \phi_{*}}$. Right plot: Performing this procedure for all $\theta_{1} \in[0, \sqrt{2} / 2]$ traces out the boundary of $\Theta_{L}$, shown as a solid line, along with the leftmost Ritz value computed from 10,000 randomly generated complex two-dimensional subspaces.

illustrates the procedure for determining points on the boundary of $\Theta_{L}$ by finding the largest $\phi$ such that $\operatorname{Re}\left(\theta_{1} e^{i \phi}\right) \geq \operatorname{Re}\left(\theta_{2} e^{i \phi}\right)$ for all valid $\theta_{2}$.

4. Conclusions. The analysis presented here bounds Ritz values from general subspaces. The bounds are a consequence of the interlacing properties of Hermitian matrices, and they provide greater insight into the interior structure of the numerical range of a matrix. By analyzing one specific matrix in detail, we have shown the subtle behavior that Ritz values of nonnormal matrices can exhibit. We have been motivated to explore these questions in order to better understand the performance of iterative methods for large-scale linear systems and eigenvalue problems. Many such methods draw approximations from Krylov subspaces. Do the Ritz values drawn from Krylov subspaces have special properties? Bujanović has recently addressed similar questions for normal matrices [2], but the nonnormal case remains to be investigated. Iterative eigensolvers repeatedly refine the approximation subspace to better align with an invariant subspace. We would like to understand how the Ritz values evolve under each step of this subspace refinement. For Hermitian matrices, interlacing provides the answer. The general case remains a challenging problem.

Acknowledgments. We thank Dan Sorensen for his observation about the applicability of our bounds to shift-invert eigenvalue computations and several referees for their thorough reviews and helpful comments.

\section{REFERENCES}

[1] R. Bhatia, Matrix Analysis, Springer-Verlag, New York, 1997.

[2] Z. Bujanović, Krylov Type Methods for Large Scale Eigenvalue Computations, Ph.D. thesis, University of Zagreb, 2011. 
[3] R. CARden, A simple algorithm for the inverse field of values problem, Inverse Problems, 25 (2009), 115019.

[4] R. Carden, Ritz Values and Arnoldi Convergence for Non-Hermitian Matrices, Ph.D. thesis, Rice University, 2011.

[5] R. Carden and D. Hansen, Ritz Values of Normal Matrices and Ceva's Theorem, Technical report TR 11-16, Department of Computational and Applied Mathematics, Rice University, 2011.

[6] C. Chorianopoulos, P. Psarrakos, and F. Uhlig, A method for the inverse numerical range problem, Electron. J. Linear Algebra, 20 (2010), pp. 198-206.

[7] M. EMBree, The Arnoldi eigenvalue iteration with exact shifts can fail, SIAM J. Matrix Anal. Appl., 31 (2009), pp. 1-10.

[8] K. E. Gustafson And D. K. M. RaO, Numerical Range: The Field of Values of Linear Operators and Matrices, Springer-Verlag, New York, 1997.

[9] R. A. Horn and C. R. Johnson, Matrix Analysis, Cambridge University Press, Cambridge, UK, 1985.

[10] R. A. Horn and C. R. Johnson, Topics in Matrix Analysis, Cambridge University Press, Cambridge, UK, 1991.

[11] R. S. Inving, Integers, Polynomials, and Rings: A Course in Algebra, Springer-Verlag, New York, 2004.

[12] R. B. Lehoucq, D. C. Sorensen, And C. Yang, ARPACK Users' Guide: Solution of LargeScale Eigenvalue Problems with Implicitly Restarted Arnoldi Methods, SIAM, Philadelphia, 1998.

[13] S. M. Malamud, Inverse spectral problem for normal matrices and the Gauss-Lucas theorem, Trans. Amer. Math. Soc., 357 (2004), pp. 4043-4064.

[14] A. W. Marshall, I. Olkin, And B. C. Arnold, Inequalities: Theory of Majorization and Its Applications, 2nd ed., Springer, New York, 2011.

[15] B. N. Parlett, The Symmetric Eigenvalue Problem, SIAM, Philadelphia, 1998.

[16] P. J. Psarrakos and M. J. Tsatsomeros, An envelope for the spectrum of a matrix, Cen. Eur. J. Math., 10 (2012), pp. 292-302.

[17] J. F. Queiró And A. L. DuARte, On the Cartesian decomposition of a matrix, Linear Multilinear Algebra, 18 (1985), pp. 77-85.

[18] Q. I. Rahman and G. Schmeisser, Analytic Theory of Polynomials, Oxford University Press, Oxford, UK, 2002.

[19] D. C. Sorensen, Implicit application of polynomial filters in a k-step Arnoldi method, SIAM J. Matrix Anal. Appl., 13 (1992), pp. 357-385.

[20] F. UhLIG, An inverse field of values problem, Inverse Problems, 24 (2008), 055019.

Copyright $@$ by SIAM. Unauthorized reproduction of this article is prohibited. 